\title{
Clinicopathological Profile, Risk Factors, and Outcomes in Distant Metastasis from Differentiated Thyroid Cancer: An Analysis of 108 Patients from a Single Institution
}

\author{
Suganya Sekar ${ }^{1}$, Anish Jacob Cherian ${ }^{2}$, Deepak Thomas Abraham ${ }^{3}$, Antonisamy Belavendra ${ }^{4}$, Paul M Jacob ${ }^{5}$
}

\begin{abstract}
Aim and objective: To study the clinicopathological profile, risk factors, and outcomes in distant metastasis from differentiated thyroid cancer in a single institution.

Materials and methods: A retrospective review of the medical records of 1,408 consecutive patients diagnosed with differentiated thyroid carcinoma (DTC) and managed at Christian Medical College, Vellore, India from January 2004 to December 2013 was performed. There were 108 patients with distant metastasis among this cohort.

Results: We had 1,408 DTC patients treated over 10 years, among them 108 patients had distant metastasis (7.7\%). Among 1,408 patients, $1,355(96.2 \%)$ were papillary carcinoma thyroid, 49 (3.5\%) were follicular, and $4(0.3 \%)$ were Hürthle cell carcinoma. The mean age was $47.84+$ 15.4 years ranging from 13 to 75 . Age $>45$ years constitute $60.2 \%$. Among the 108 distant metastasis patients, $85.2 \%$ were papillary, and the remaining $14.8 \%$ follicular carcinoma. A significant proportion of patients had T3, T4 disease (63.9\%) and nodes involved (50\%). On logistic regression analysis, the adjusted odds ratio (OR) for detection of metastasis in this series was significantly high for age $>45$, male gender, follicular cancer or follicular variant papillary thyroid carcinoma (PTC), higher stage T3/T4 primary, and the presence of nodal disease. Of the 108 patients, $64.8 \%$ presented with metastases. Among the patients presented with metastatic symptoms, $9.3 \%$ had paraparesis or paraplegia. Isolated bone (40.7\%) is the commonest site of involvement followed by isolated lung (32.4\%) and multiple organ pattern of involvement is the least common (6.5\%). All patients with metastatic follicular carcinoma had bony metastasis either alone (81.3\%) or rarely with lung (18.7\%). The whole-body iodine scan showed positive uptake in $85.7 \%$ metastatic patients and was negative in $14.3 \%$. Metastases involving only bone was significantly associated with radioactive iodine avidity. Among the patients who underwent radioiodine therapy, $78.5 \%$ have shown a good response to iodine therapy. Patients with age 45 years or patients with bone-only or multiple distant metastatic sites. Metastasectomy was done in 13 patients, curative surgery in 7 and palliative in 6.

Conclusion: The prevalence of distant metastasis among our series of DTC is $7.7 \%$ with a higher proportion from follicular carcinoma. Significant numbers of patients have distant metastases during the initial evaluation of the primary and it can have a quite morbid presentation like paraparesis and paraplegia in some patients. Bone is the most preferred site of metastases, which requires more studies to evaluate the cause especially in an iodine-deficient country like India. It is possible to treat distant metastases effectively with radioactive iodine therapy as considerable responses are present in most of the patients. Improved efficacy of radioiodine therapy is present in younger patients and for lung metastases. Metastasectomy especially for solitary skeletal metastases is a considerable option.

Clinical significance: The pattern of disease in India-the iodine deficiency soil, is different compared to other parts of the world. With the possibility to treat with radioiodine and surgery, extended survival is a possibility in metastatic thyroid disease. When the majority of patients with metastases are diagnosed at presentation, sometimes even presenting with paraplegia, public awareness should be raised to avoid neglecting the thyroid illness.

Keywords: Differentiated thyroid cancer, Metastasectomy, Radioiodine therapy, Thyroid metastases.

World Journal of Endocrine Surgery (2020): 10.5005/jp-journals-10002-1296
\end{abstract}

\section{INTRODUCTION}

The incidence of differentiated thyroid carcinoma (DTC) though reportedly showing a steady rise worldwide, is large of good prognosis. ' Distant metastasis in DTC is uncommon reportedly presenting in the range of $1.6-22 \% .^{2-5}$ However, it has a significant impact on prognosis ${ }^{6}$ as evidenced by a 10 -year survival of $40 \%$ for patients with metastatic DTC compared to $80-95 \%$ in patients with non-metastatic DTC. ${ }^{7}$ There are limited data from the Indian subcontinent; our institution receives patients from a large area of the subcontinent, so we analyzed our experience with the presentation of this disease and outcomes.

\section{Materials and Methods}

\section{Study Population}

A retrospective review of the medical records of 1,408 consecutive patients diagnosed with DTC and managed at Christian Medical
1-3,5 Department of Endocrine Surgery, Christian Medical College, Vellore, Tamil Nadu, India

${ }^{4}$ Department of Biostatistics, Christian Medical College, Vellore, Tamil Nadu, India

Corresponding Author: Suganya Sekar, Department of Endocrine Surgery, Christian Medical College, Vellore, Tamil Nadu, India, Phone: +919894767376, e-mail: drsugancbe@gmail.com

How to cite this article: Sekar S, Cherian AJ, Abraham DT, et al. Clinicopathological Profile, Risk Factors, and Outcomes in Distant Metastasis from Differentiated Thyroid Cancer: An Analysis of 108 Patients from a Single Institution. World J Endoc Surg 2020;12(2): 54-59.

Source of support: Nil

Conflict of interest: None

(c) The Author(s). 2020 Open Access This article is distributed under the terms of the Creative Commons Attribution 4.0 International License (https://creativecommons. org/licenses/by-nc/4.0/), which permits unrestricted use, distribution, and non-commercial reproduction in any medium, provided you give appropriate credit to the original author(s) and the source, provide a link to the Creative Commons license, and indicate if changes were made. The Creative Commons Public Domain Dedication waiver (http://creativecommons.org/publicdomain/zero/1.0/) applies to the data made available in this article, unless otherwise stated. 
College, Vellore, India from January 2004 to December 2013 was performed. There were 108 patients with distant metastasis among this cohort.

\section{Methods}

The study was approved by our institutional review board. The medical records were reviewed from charts and the electronic database. The demographic data, clinicopathological profile, operative, radio ablation details, and follow-up findings were collected. For patients who defaulted treatment, follow-up was carried out through telephonic conversation or postal correspondence to acquire details of health status and outcome.

\section{Treatment of Thyroid Primary and Metastatic Disease}

Our institution protocol for the initial surgical management of DTC confined to the thyroid during the study period was total thyroidectomy with central/lateral neck dissection for cervical lymph nodes that are clinically, radiologically, or intraoperatively suspicious selectively confirmed by cytology or frozen section. We did not perform prophylactic central dissection but assess the pre- and paratracheal groups of nodes during thyroidectomy for proven or suspected well differentiated thyroid carcinoma (WDTC) with therapeutic dissection for involved nodes. This was followed by a whole-body radioiodine diagnostic scan (WBS) with $70 \mathrm{MBq}$ ( $2 \mathrm{mCi}$ ) of ${ }^{131}$ I to detect residual and/or metastatic disease. Patients with a positive WBS receive ${ }^{131}$ I therapy; the dosage given was 1,110 to $1,850 \mathrm{MBq}(30-50 \mathrm{mCi}$ ) for thyroid remnant, 1,850 to $3,700 \mathrm{MBq}$ (50-100 mCi) for regional lymph nodes, and 5,550 MBq (100-150) for distant metastasis. The WBS was repeated at 6-month intervals until two consecutive scans were negative. lodine avid remnants and metastatic lesions were ablated. Response to radioiodine therapy was assessed by serum thyroglobulin level and the radioiodine uptake characteristics and accordingly categorized as progression, regression, stable, or complete resolution. Surgery for a metastatic lesion in the bone was performed if they were solitary (curative intent) or unstable to relieve spinal cord compression, fracture of long bones (symptom palliation).

\section{Follow-up}

All patients were followed up by clinical examination and estimation of serum thyroglobulin levels and selective use of ultrasound imaging. Serum thyroglobulin was measured using a chemiluminescent assay. In patients with an elevated thyroglobulin level and negative radioiodine WBS and ultrasound, other imaging modalities, such as, computed tomography (CT) scan and PET scan were performed as per the clinical setting to detect persistent, recurrent, or metastatic lesions to assess the structural disease. Management was individualized with selective use of surgical excision, local radiation therapy, or tyrosine kinase inhibitors.

\section{Statistical Analysis}

Categorical variables were presented as frequencies and relative frequencies. Continuous variables were summarized with appropriate measures of central tendency and dispersion. In crude analysis (unadjusted), we calculated the OR and their 95\% confidence intervals (Cls) associated with each risk factor. Adjusted OR and their $95 \% \mathrm{Cls}$ were estimated using multiple logistic regression analysis. We determined variables significantly associated with distant metastasis at a significance level of 0.05 .
Data were analyzed using Stata version 13.0 (StataCorp. 2013. Stata Statistical Software: Release 13. College Station, Texas, USA: StataCorp LP).

\section{RESULTS}

We had 1,408 DTC patients treated over 10 years, among them 108 patients had distant metastasis (7.7\%). Among 1,408 patients, 1,355 (96.2\%) were papillary carcinoma thyroid, 49 (3.5\%) were follicular and $4(0.3 \%)$ were Hürthle cell carcinoma. Among the papillary carcinoma, 905 (66.8\%) were classical type, 435 (32.1\%) were of follicular variant, $3(0.2 \%)$ each of diffuse sclerosing, columnar and oxyphil variants, $4(0.3 \%)$ tall cell, $1(0.07 \%)$ each of nodular fasciitis and hobnail variants.

In this series, the detection of metastases in papillary carcinoma thyroid was only $6.8 \%$ as compared to follicular which was $32.6 \%$. We had four Hürthle cell carcinoma patients but none had detectable systemic metastasis.

\section{Presentation of Metastases and Clinical Characteristics of Metastases}

Of the 108 patients, $64.8 \%$ presented with metastases, $22.2 \%$ was diagnosed by the first whole-body iodine scan around 3-6 months after surgery, $13 \%$ developed metastases during follow-up between 13 months and 204 months after surgery with an average of 51 months and a median of 36 months. The metastases in $13 \%^{8}$ patients were detected by whole-body iodine scan in 7 patients, $\mathrm{X}$-ray long bones in 2 patients, magnetic resonance imaging (MRI) thigh and hip (suspected as sarcoma) in 1 patient, CT thorax for lungs in 2 patients, CT brain in 1 patient, and fluorodeoxyglucose positron emission tomography (FDG PET) in 1 patient.

The mean age was $47.84 \pm 15.4$ years ranging from 13 to 75 . Age $>45$ years constitute $60.2 \%$. Among the 108 distant metastasis patients, $85.2 \%$ were papillary, and the remaining $14.8 \%$ follicular carcinoma. A significant proportion of patients had T3, T4 disease (63.9\%) and nodes involved (50\%) (Table 1). On logistic regression analysis, adjusted OR for detection of metastasis in this series was significantly high for age $>45$, male gender, follicular cancer or follicular variant papillary thyroid carcinoma (PTC), higher stage T3/T4 primary, and the presence of nodal disease.

\section{Site and Type of Involvement}

Isolated bone is the commonest site of involvement followed by isolated lung and multiple organ pattern of involvement is the least common. The majority (78.6\%) of the cases with multiple site involvement had bone with lung metastases.

Bone metastasis was more likely to be multiple (66.2\%) compared to solitary (33.8\%). Among the 68 patients with bone metastasis, 39 (57.4\%) patients had vertebral involvement making it the commonest site. We had a single patient with only brain metastasis, two patients with brain and bone involved, and one with brain and lung metastasis.

Interestingly, we had a patient with a brain together with the involvement of the breast which is a rare site of metastasis. All the brain metastases patients happened to be papillary variant.

All patients with metastatic follicular carcinoma had bony metastasis either alone (81.3\%) or rarely with lung (18.7\%). Most $(14 / 16)$ were aged $>45$ years $(87.5 \%)$. Patients aged $<45$ years predominantly $(95.2 \%)$ had papillary carcinoma, more than half of these patients (51.2\%) had only lung metastasis, however, about $33.7 \%$ had only bone metastases (Table 2 ). 
Table 1: Logistic regression analysis for clinical characteristics associated with distant metastasis

\begin{tabular}{|c|c|c|c|c|c|c|}
\hline \multirow[b]{2}{*}{ Characteristics } & \multirow[b]{2}{*}{$n=108$} & \multirow[b]{2}{*}{$\%$} & \multicolumn{2}{|c|}{ Crude } & \multicolumn{2}{|c|}{ Adjusted } \\
\hline & & & OR $(95 \% \mathrm{Cl})$ & $p$ value & OR $(95 \% \mathrm{Cl})$ & p value $e^{t}$ \\
\hline \multicolumn{7}{|l|}{ Age (years) } \\
\hline$\leq 45$ (ref) & 43 & 39.8 & 1.0 & & 1.0 & \\
\hline$>45$ & 65 & 60.2 & $3.2(2.2-4.8)$ & $<0.001$ & $2.7(1.6-4.4)$ & $<0.001$ \\
\hline \multicolumn{7}{|l|}{ Gender } \\
\hline Male & 48 & 44.4 & $1.9(1.3-2.8)$ & 0.002 & $1.2(0.7-2.0)$ & 0.52 \\
\hline Female (ref) & 60 & 55.6 & & & & \\
\hline \multicolumn{7}{|l|}{ Pathology } \\
\hline Papillary Ca-Classical (ref) & 51 & 47.2 & 1.0 & & 1.0 & \\
\hline Papillary Ca-Follicular variant & 40 & 37.1 & $1.7(1.1-2.6)$ & 0.02 & $4.7(2.4-9.3)$ & $<0.001$ \\
\hline Papillary Ca_Other variants (diffuse sclerosing) & 1 & 0.9 & $0.98(0.1-7.6)$ & 0.99 & $1.6(0.1-16.6)$ & 0.71 \\
\hline Follicular Ca & 16 & 14.8 & $8.1(4.2-15.7)$ & $<0.001$ & $17.3(6.4-46.8)$ & $<0.001$ \\
\hline \multicolumn{7}{|l|}{ T stage } \\
\hline T1 (ref) & 5 & 4.6 & 1.0 & & 1.0 & \\
\hline $\mathrm{T} 2$ & 15 & 13.9 & $3.0(1.1-8.4)$ & 0.04 & $2.5(0.9-7.1)$ & 0.08 \\
\hline T3 & 34 & 31.5 & $5.9(2.3-15.4)$ & $<0.001$ & $4.3(1.6-11.4)$ & 0.003 \\
\hline T4 & 35 & 32.4 & $74.9(27.4-204.1)$ & $<0.001$ & $52.7(18.1-153.3)$ & $<0.001$ \\
\hline Tx & 19 & 17.6 & & & & \\
\hline \multicolumn{7}{|l|}{$\mathrm{N}$ stage } \\
\hline NO (ref) & 52 & 48.1 & 1.0 & & 1.0 & \\
\hline N1 & 54 & 50 & $2.5(1.7-3.7)$ & $<0.001$ & $3.8(1.9-7.3)$ & $<0.001$ \\
\hline $\mathrm{Nx}$ & 2 & 1.9 & & & & \\
\hline
\end{tabular}

${ }^{\dagger}$ Covariates included in the adjusted model: age, gender, pathology, T stage, and $\mathrm{N}$ stage

Table 2: Pathological types and the distant metastasis site

\begin{tabular}{|c|c|c|c|c|c|}
\hline \multirow[b]{2}{*}{ Metastatic sites $(n=108)$} & \multicolumn{2}{|c|}{ Papillary carcinoma $(n=92)$} & \multicolumn{2}{|c|}{ Follicular carcinoma $(n=16)$} & \multirow[b]{2}{*}{ Total } \\
\hline & Age $\leq 45$ years & Age $>45$ years & Age $\leq 45$ years & Age $>45$ years & \\
\hline Bone only & $8(25.8 \%)$ & $23(74.2 \%)$ & $2(15.4 \%)$ & $11(84.6 \%)$ & $44(40.7 \%)$ \\
\hline Lung only & $22(62.9 \%)$ & $13(37.1 \%)$ & 0 & 0 & $35(32.4 \%)$ \\
\hline Bone and lung & $7(29.2 \%)$ & $17(70.8 \%)$ & 0 & $3(100 \%)$ & $22(20.4 \%)$ \\
\hline $\begin{array}{l}\text { Other sites including brain, soft } \\
\text { tissue, and breast }\end{array}$ & $3(42.9 \%)$ & $4(57.1 \%)$ & 0 & 0 & $7(6.5 \%)$ \\
\hline
\end{tabular}

\section{Symptom at Presentation and Diagnostic Imaging Modality of Metastases}

Patients symptomatic at the time of diagnosis of metastases were 62 (57.4\%), with pain along with or without bony swelling in $26.9 \%$, painless bony swelling in $8.3 \%$, vertebral metastases with motor symptoms like paraparesis or paraplegia in $9.3 \%$, respiratory symptoms like cough, hemoptysis, and dyspnea in $12.9 \%$. Imaging modalities like X-ray, CT scan, and MRI, whole-body iodine scan all almost equally contributed to the diagnosis (27, 25.9, 17, and 30\%). PET scan was used for diagnosis in only one patient who had rising serum thyroglobulin and negative radioactive iodine scan.

\section{Surgery for Primary}

Total or completion thyroidectomy with $(48.1 \%)$ or without node dissection (45.4\%) was done in $93.5 \%$. Debulking thyroidectomy was carried out for inoperable tumors by $6.5 \%$.

\section{Radioactive lodine Therapy}

A whole-body iodine scan was done in 91 (84.3\%) patients at our institute, with no follow-up data on 17 patients who were probably treated closer to home. Median stimulated serum thyroglobulin done before the first whole-body iodine scan was $1,420 \mathrm{ng} / \mathrm{mL}$. The whole-body iodine scan showed positive uptake in $85.7 \%$ metastatic patients and was negative in $14.3 \%$. Various factors associated with radioactive iodine avidity was analyzed, metastases involving only bone was significantly associated with radioactive iodine avidity (Table 3). All the patients with follicular carcinoma and $83.5 \%$ of papillary carcinoma metastases had positive radioactive iodine uptake.

Among the papillary carcinomas, the follicular variant had more positive uptake (31/32) than the classical variant (34/46), the association was significant $p=0.01$.

Radioactive iodine therapy was administered to all patients with positive uptake and also empirically to patients with elevated thyroglobulin with negative uptake. The response to radioactive iodine therapy could be assessed in 65 patients who continued treatment. Among the 65 patients, $78.5 \%$ have shown response to iodine therapy in the form of regression, complete response, or at least stable disease but the remaining $21.5 \%$ had progression of the disease. The median number of radioactive iodine therapy doses 
Clinicopathological Profile, Risk Factors, and Outcomes in Distant Metastasis from DTC

Table 3: Radioactive iodine avidity (positive vs negative uptake) and associated variables using logistic regression analysis

\begin{tabular}{|c|c|c|c|c|c|c|}
\hline \multirow[b]{2}{*}{ Variables } & \multirow[b]{2}{*}{ Positive, $n$ (\%) } & \multirow[b]{2}{*}{ Negative, $n$ (\%) } & \multicolumn{2}{|c|}{ Crude } & \multicolumn{2}{|c|}{ Adjusted } \\
\hline & & & OR $(95 \% \mathrm{Cl})$ & $p$ value & OR $(95 \% \mathrm{Cl})$ & $p$ value \\
\hline \multicolumn{7}{|l|}{ Age (years) } \\
\hline$\leq 45$ & $34(89.5)$ & $4(10.5)$ & $1.7(0.5-6.1)$ & 0.39 & $2.9(0.7-11.5)$ & 0.13 \\
\hline$>45$ & $44(83.0)$ & $9(17.0)$ & & & & \\
\hline \multicolumn{7}{|l|}{ Pathological type } \\
\hline Follicular & $12(100.0)$ & $0(0.0)$ & & - & & - \\
\hline Papillary & $66(83.5)$ & $13(16.5)$ & & & & \\
\hline \multicolumn{7}{|l|}{ Metastatic site } \\
\hline Bone only & $33(97.1)$ & $1(2.9)$ & $11(1.2-98.6)$ & 0.03 & $14.1(1.5-136.5)$ & 0.02 \\
\hline Lung only & $27(81.8)$ & $6(18.2)$ & $1.5(0.4-5.4)$ & 0.53 & $1.2(0.3-4.6)$ & 0.80 \\
\hline Multiple sites & $18(75.0)$ & $6(25.0)$ & & & & \\
\hline
\end{tabular}

$\mathrm{OR}$, odds ratio; $\mathrm{Cl}$, confidence interval

Table 4: Response to radioiodine therapy and associated factors

\begin{tabular}{|c|c|c|c|c|c|}
\hline \multirow[b]{2}{*}{ Variables } & \multicolumn{4}{|c|}{ Response to radioactive iodine therapy } & \multirow[b]{2}{*}{$p$ value } \\
\hline & Progression, $n(\%)$ & Regression, $n$ (\%) & Static, $n(\%)$ & Resolution, $n(\%)$ & \\
\hline \multicolumn{6}{|l|}{ Age (years) } \\
\hline$\leq 45$ & $1(4 \%)$ & $16(64 \%)$ & $4(16 \%)$ & $4(16 \%)$ & $<0.03$ \\
\hline$>45$ & $13(32.5 \%)$ & $16(40 \%)$ & $8(20 \%)$ & $3(7.5 \%)$ & \\
\hline \multicolumn{6}{|l|}{ Pathological type } \\
\hline Follicular & $4(40 \%)$ & $3(30 \%)$ & $3(30 \%)$ & 0 & $<0.18$ \\
\hline Papillary & $10(18.2 \%)$ & $29(52.7 \%)$ & $9(16.4 \%)$ & $7(12.7 \%)$ & \\
\hline \multicolumn{6}{|l|}{ Site of metastasis } \\
\hline Bone only & $8(27.6 \%)$ & $14(48.3 \%)$ & $7(24.1 \%)$ & 0 & \\
\hline Lung only & 0 & $13(59.1 \%)$ & $4(18.2 \%)$ & $5(22.7 \%)$ & $<0.01$ \\
\hline Multiple sites & $6(42.9 \%)$ & $5(35.7 \%)$ & $1(7.1 \%)$ & $2(14.3 \%)$ & \\
\hline
\end{tabular}

was one in the complete response group, three in the regression and progression group, and four in the stable group. Among the 65 patients, empirical iodine therapy was administered for 9 patients whose scan was negative, of whom regression was seen in 3 , stability in 1, and progression in 5 based on thyroglobulin levels.

We analyzed the factors affecting the response to radioactive iodine therapy. A significant association between age, site of metastasis, and response to therapy was present. Patients with age $\leq 45$ years, metastasis in lung alone had a significantly better response to iodine therapy compared to patients aged $>45$ years or patients with bone-only or multiple distant metastatic sites as shown in Table 4.

\section{Surgery for Metastases}

Metastasectomy was done in 13 patients, curative surgery in 7 and palliative in 6 . Vertebral metastases resection was done in five patients-laminectomy and partial excision of tumor in three, en bloc excision of sacral metastases in one, and L5 S1 vertebra in another patient. In the long bones, palliative fixation of fracture femur was done in two patients, excision of clavicular metastases in one and en bloc excision with prosthesis fixation of the humerus in one patient. One patient underwent disarticulation of the hip for resection of femoral metastases which was misdiagnosed as sarcoma. Diagnostic resection of brain metastases was performed in one patient. One patient underwent excision of tumor in the skin and subcutaneous tissue of the neck and the other patient had her breast metastases excised.

\section{External Beam Radiation Therapy (EBRT)}

Fifteen patients received external beam radiation therapy (EBRT). Radiation therapy (RT) as adjuvant treatment was administered for three patients after spine surgery, one patient after excision of humeral metastasis. Radiation therapy for brain metastasis was given for one patient. Three patients with paraparesis received RT to relieve spinal cord compression. Radiation therapy to palliate pain was given to four patients with vertebral metastases and three with pelvic metastases.

\section{Follow-up and Survival}

Follow-up details were available in 91 patients. The median follow-up was 39 months. We observed mortality in 10 (11\%) patients. The cause of death was known in five patients, four of them died of respiratory failure due to lung metastases, and one patient died of pulmonary embolism unrelated to the disease. Five of the 13 who underwent metastasectomy were lost to follow-up; the remaining 8 patients had a median follow-up of 55 months. Among them, only one patient expired after 60 months of metastasectomy. Radioiodine scan was positive in six patients and negative in two patients, all eight patients had radioiodine therapy. The 5-year survival rate for the metastases patients as a whole was $86.2 \%$ by Kaplan-Meier survival estimate (Fig. 1).

\section{Discussion}

The reported prevalence of distant metastases in WDTC varies from 1.6 to $22 \%(2-5)$, in our series it is $7.7 \%$. Our prevalence of 


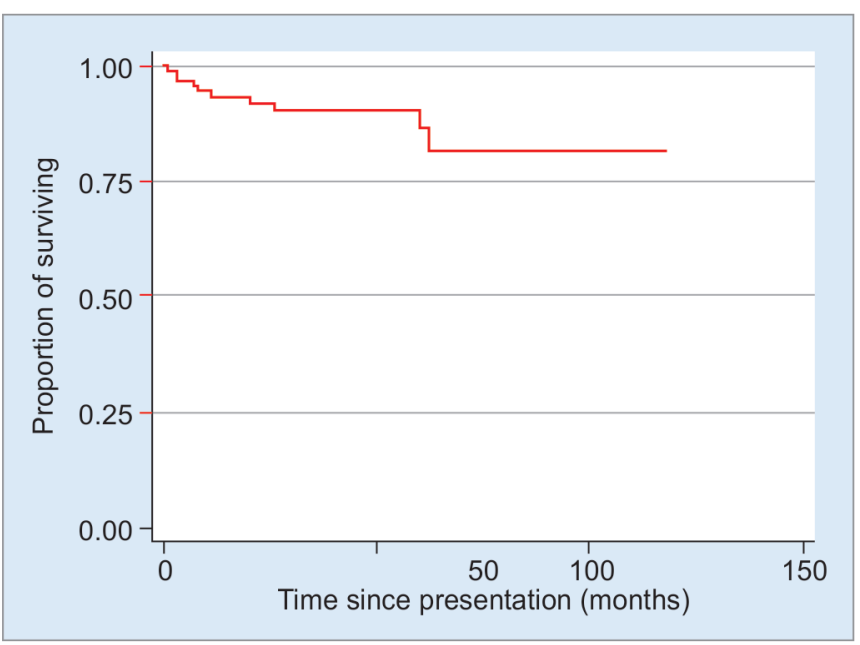

Fig. 1: Survival curve

follicular carcinoma metastases is $32.6 \%$ which compares with $40 \%$ from another series in our region reported by Mishra et al. ${ }^{2}$ and is higher than other series by Benbassat et al. $21 \%$ and Shaha et al. $12.2 \% .^{4,9}$ Only two other studies ${ }^{2,3}$ report $>80 \%$ of metastatic disease detected at presentation including the first iodine scan, whereas four other of the studies have $<80 \%$; $4,10-12$ this may reflect the delayed presentation of disease due to socioeconomic factors.

Age $>45$ years is an important prognostic factor of poor survival, ${ }^{5,6,13,14}$ and most of our patients (60.2\%) who were aged $>45$ years presented with advanced tumors (T3/T4, N1) 63\%, as also reported by other authors. ${ }^{2,5,8,14}$ Goffredo et al. ${ }^{13}$ and Clark et al. ${ }^{11}$ have also substantiated by analyzing in comparison with the non-metastatic group that older age group, male sex, larger tumor size $(>4 \mathrm{~cm})$, extrathyroidal disease, and nodal metastases are all predictive factors for metastases.

Thyroid carcinoma commonly metastasizes to lung and bone, with the lung being the commonest site in the international literature. ${ }^{5,6,8,10,14-16}$ Interestingly, contrary to all other series, bone is the commonest site of involvement $40.7 \%$ in our study, and in one other study by Mishra et al. ${ }^{2}$ from India. This may be due to a referral bias for more symptomatic disease with a higher rate of follicular and follicular variant of papillary carcinoma which has a predilection to metastasize to the bone; follicular component formed $51 \%$ of total pathological types in our study. Most of the follicular carcinoma metastases were confined to bone-only (81.3\%) and in older patients $>45$ years $(52.3 \%)$ compared to lung only metastases (38\%) and younger patients $(51.2 \%)$ in papillary type, similar rates are reported by Durante et al. ${ }^{15}$ and Mishra et al., ${ }^{2}$ but series by Benbassat et al. ${ }^{4}$ has published no association between metastatic site and age. A vertebra is a common site of skeletal metastases in thyroid cancer. ${ }^{12}$ It is also reported that multiple skeletal metastases are associated with poor survival. ${ }^{17}$ We have $66.2 \%$ of our multiple bony metastases.

More than half of our patients as a whole including metastases to all sites are symptomatic often presenting with bony pain, in $9.3 \%$ it is even worse as they presented with paraparesis and paraplegia, the rate of which is higher than in other reports, ${ }^{2-4,10,17}$ although some of the authors studied only bone metastases. 7,17

Total thyroidectomy which is important to facilitate radioactive iodine treatment was possible in $92.3 \%$ of our patients. Radioiodine therapy is an effective systemic treatment and is known to improve survival especially if the metastases are iodine avid. ${ }^{3,5,10,12,15,17,18}$ The well-differentiated nature of the tumor favors its excellent uptake of iodine in most patients ranging from 64 to $88 \%$ in various series, ${ }^{10,12,14-16}$ even in the current study it is $85.7 \%$. We noted response to radioiodine treatment by assessing activity in radioiodine scan and change in serum thyroglobulin. We have $78.5 \%$ showing good response in the form of regression, static, resolution of the disease. Assessment of response to ${ }^{131}$ I therapy is different in various studies. Schlumberger et al. ${ }^{16}$ assessed response by evidence of disease in either radiographs or iodine scan, $31.5 \%$ had a complete response at the end of the study. Durante et al. ${ }^{15}$ measured disease activity in bone metastases by the presence or absence of uptake in iodine scan, $43 \%$ of them had achieved remission. Qiu et al. ${ }^{17}$ had considered a change in serum thyroglobulin, resolution of pain, anatomical imaging changes as a marker of the response of bone metastases to ${ }^{131}$ I therapy, in their report $34.9 \%$ of patients had a decrease in serum thyroglobulin and in $52.8 \%$ it was stable, $63.9 \%$ had significant pain relief, $76.4 \%$ had no obvious change in anatomical imaging.

Factors influencing the avidity and treatment response to ${ }^{131}$ I therapy are analyzed. We have follicular carcinoma and metastases confined to only bone showing good uptake, age was not a significant factor but younger patients found to have good uptake in most studies s $^{3,14,16,19}$ and site of metastases was not found to affect uptake in the series by Shoup et al. ${ }^{6}$ and Durante et al. ${ }^{15}$ Younger patients and lung only metastases have a good response to radioiodine treatment in our study, corresponding results are published by Durante et al. ${ }^{15}$

Control of pain can be significantly achieved by EBRT or metastasectomy, the latter has also been shown to improve survival especially if it is oligometastases or preferably solitary. $710,15,17,20,21$ Curative surgery as compared to palliative influence survival rate. ${ }^{710,12,21}$ In our study, curative surgery was possible in 7 among the 13 metastasectomy patients. The 5 -year survival rate in the present study is comparable to the series by Kim et al. $^{3}$ and Benbassat et al. ${ }^{4}$

The limitation of the study is that it is a retrospective study, longer survival rates could not be commented on because of limited follow-up. Long-term benefits of curative metastasectomy or outcome due to other modalities of treatment like systemic therapy with molecular targets in progressive disease could not be assessed as they are of recent practice.

\section{Conclusion}

The prevalence of distant metastasis among our series of DTC is $7.7 \%$ with a higher proportion from follicular carcinoma. Significant numbers of patients have distant metastases during the initial evaluation of the primary and it can have a quite morbid presentation like paraparesis and paraplegia in some patients. Bone is the most preferred site of metastases, which requires more studies to evaluate the cause especially in an iodine-deficient country like India. It is possible to treat distant metastases effectively with radioactive iodine therapy as considerable responses are present in most of the patients. Improved efficacy of radioiodine therapy is present in younger patients and for lung metastases. Metastasectomy especially for solitary skeletal metastases is a considerable option. 


\section{References}

1. Haugen BR, Alexander EK, Bible KC, et al. 2015 American Thyroid Association Management guidelines for adult patients with thyroid nodules and differentiated thyroid cancer. Thyroid Off J Am Thyroid Assoc 2016;26(1):1-133. DOI: 10.1089/thy.2015.0020.

2. Mishra A, Mishra SK, Agarwal A, et al. Metastatic differentiated thyroid carcinoma: clinicopathological profile and outcome in an iodine deficient area. World J Surg 2002;26(2):153-157. DOI: 10.1007/ s00268-001-0198-x.

3. Kim HJ, Lee Jl, Kim NK, et al. Prognostic implications of radioiodine avidity and serum thyroglobulin in differentiated thyroid carcinoma with distant metastasis. World J Surg 2013;37(12):2845-2852. DOI: 10.1007/s00268-013-2213-4.

4. Benbassat CA, Mechlis-Frish S, Hirsch D. Clinicopathological characteristics and long-term outcome in patients with distant metastases from differentiated thyroid cancer. World J Surg 2006;30(6):1088-1095. DOI: 10.1007/s00268-005-0472-4.

5. Mihailovic J, Stefanovic L, Malesevic M. Differentiated thyroid carcinoma with distant metastases: probability of survival and its predicting factors. Cancer Biother Radiopharm 2007;22(2):250-255. DOI: $10.1089 / \mathrm{cbr} .2006 .313$.

6. Shoup M, Stojadinovic A, Nissan A, et al. Prognostic indicators of outcomes in patients with distant metastases from differentiated thyroid carcinoma. J Am Coll Surg 2003;197(2):191-197. DOI: 10.1016/ S1072-7515(03)00332-6.

7. Zettinig G, Fueger BJ, Passler C, et al. Long-term follow-up of patients with bone metastases from differentiated thyroid carcinoma -- surgery or conventional therapy? Clin Endocrinol (Oxf) 2002;56(3):377-382. DOI: 10.1046/j.1365-2265.2002. 01482.x.

8. Haq M, Harmer C. Differentiated thyroid carcinoma with distant metastases at presentation: prognostic factors and outcome. Clin Endocrinol (Oxf) 2005;63(1):87-93. DOI: 10.1111/j.13652265.2005.02304.x.

9. Shaha $A R$, Ferlito A, Rinaldo A. Distant metastases from thyroid and parathyroid cancer. ORL J Oto-Rhino-Laryngol Its Relat Spec 2001;63(4):243-249. DOI: 10.1159/000055749.

10. Lee J, Soh E-Y. Differentiated thyroid carcinoma presenting with distant metastasis at initial diagnosis clinical outcomes and prognostic factors. Ann Surg 2010;251(1):114-119. DOI: 10.1097/ SLA.0b013e3181b7faf6.
11. Clark JR, Lai P, Hall F, et al. Variables predicting distant metastases in thyroid cancer. Laryngoscope 2005;115(4):661-667. DOI: 10.1097/01. mlg.0000161337.46892.e0.

12. Bernier MO, Leenhardt L, Hoang C, et al. Survival and therapeutic modalities in patients with bone metastases of differentiated thyroid carcinomas. J Clin Endocrinol Metab 2001;86(4):1568-1573. DOI: 10.1210/jcem.86.4.7390.

13. Goffredo P, Sosa JA, Roman SA. Differentiated thyroid cancer presenting with distant metastases: a population analysis over two decades. World J Surg 2013;37(7):1599-1605. DOI: 10.1007/s00268013-2006-9.

14. Nixon IJ, Whitcher MM, Palmer FL, et al. The impact of distant metastases at presentation on prognosis in patients with differentiated carcinoma of the thyroid gland. Thyroid Off J Am Thyroid Assoc 2012;22(9):884-889. DOI: 10.1089/thy.2011.0535.

15. Durante C, Haddy N, Baudin E, et al. Long-term outcome of 444 patients with distant metastases from papillary and follicular thyroid carcinoma: benefits and limits of radioiodine therapy. J Clin Endocrinol Metab 2006;91(8):2892-2899. DOI: 10.1210/jc.2005-2838.

16. Schlumberger $M$, Challeton $C$, De Vathaire $F$, et al. Radioactive iodine treatment and external radiotherapy for lung and bone metastases from thyroid carcinoma. J Nucl Med Off Publ Soc Nucl Med 1996;37(4):598-605.

17. Qiu Z-L, Song H-J, Xu Y-H, et al. Efficacy and survival analysis of ${ }^{131}$ I therapy for bone metastases from differentiated thyroid cancer. J Clin Endocrinol Metab 2011;96(10):3078-3086. DOI: 10.1210/jc.2011-0093.

18. Higashi T, Nishii R, Yamada $S$, et al. Delayed initial radioactive iodine therapy resulted in poor survival in patients with metastatic differentiated thyroid carcinoma: a retrospective statistical analysis of 198 cases. J Nucl Med Off Publ Soc Nucl Med 2011;52(5):683-689. DOI: $10.2967 /$ jnumed.110.081059.

19. Ronga $G$, Filesi $M$, Montesano $T$, et al. Lung metastases from differentiated thyroid carcinoma. A 40 years' experience. Q J Nucl Med Mol Imaging. 2004;48(1):12-19.

20. Orita $Y$, Sugitani I, Matsuura M, et al. Prognostic factors and the therapeutic strategy for patients with bone metastasis from differentiated thyroid carcinoma. Surgery 2010;147(3):424-431. DOI: 10.1016/j.surg.2009.10.009.

21. Nakayama R, Horiuchi K, Susa M, et al. Clinical outcome after bone metastasis (BM) surgery in patients with differentiated thyroid carcinoma (DTC): a retrospective study of 40 cases. Jpn J Clin Oncol 2014;44(10):918-925. DOI: 10.1093/jjco/hyu099. 